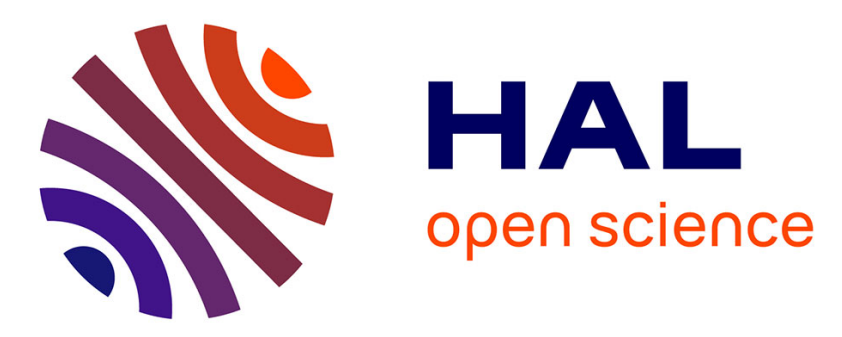

\title{
tail behavior of a threshold autoregressive stochastic volatility model
}

\author{
Aliou Diop, Dominique Guegan
}

\section{To cite this version:}

Aliou Diop, Dominique Guegan. tail behavior of a threshold autoregressive stochastic volatility model.

Extremes, 2005, 7, pp.369 - 377. halshs-00188530

\section{HAL Id: halshs-00188530 \\ https://shs.hal.science/halshs-00188530}

Submitted on 17 Nov 2007

HAL is a multi-disciplinary open access archive for the deposit and dissemination of scientific research documents, whether they are published or not. The documents may come from teaching and research institutions in France or abroad, or from public or private research centers.
L'archive ouverte pluridisciplinaire $\mathbf{H A L}$, est destinée au dépôt et à la diffusion de documents scientifiques de niveau recherche, publiés ou non, émanant des établissements d'enseignement et de recherche français ou étrangers, des laboratoires publics ou privés. 


\title{
Tail behavior of a Threshold Autoregressive Stochastic Volatility model
}

\author{
Aliou DIOP* \\ Dominique GUEGAN ${ }^{\dagger}$
}

October 21, 2007

\begin{abstract}
We consider a threshold autoregressive stochastic volatility model where the driving noises are sequences of iid regularly random variables. We prove that both the right and the left tails of the marginal distribution of the log-volatility process $\left(\alpha_{t}\right)_{t}$ are regularly varying with tail exponent $-\alpha$ with $\alpha>0$. We also determine the exact values of the coefficients in the tail behaviour of the process $\left(\alpha_{t}\right)_{t}$.

keywords : Tail Behavior, Heavy Tail, Stochastic Volatility Model, Threshold Autoregressive Model.
\end{abstract}

\section{Introduction}

Modelling the volatility of financial returns has been the purpose of many investigations. See Clark (1973), Nelson (1991), Taylor (1986, 1994), Andersen (1994), and others. There exists a lot of versions of stochastic volatility models in the literature. Here we are interested in a discrete time version of volatility model introduced first by Taylor (1986). This model appears as a particular model of the Stochastic Autoregressive Volatility (SARV) model introduced by Andersen (1994)

However the SARV model remains limited. This model does not take into account the leverage effect and the heavy tail behavior often encountered in financial returns. An alternative specification is to allow the log-volatility process

*LERSTAD, B.P. 234, UFR de Sciences Appliquées et de Technologies, Université Gaston Berger, Saint-Louis, Sénégal, e-mail : alioudiop52@yahoo.fr

${ }^{\dagger}$ E.N.S. Cachan, Equipe MORA, IDHE UMR CNRS C8533, 61 Avenue du Président Wilson, 94235, Cachan Cedex, France, e-mail : dominique.guegan@ecogest.ens-cachan.fr 
to switch between two first-order autoregressive processes with heavy tailed innovations. The use of linear processes with heavy tailed innovations is not new in time series modelling. Examples where such models appear to be appropriate have been found by Stuck and Kleiner (1974), who considered telephone signals, and Fama (1965), who modelled stock market prices. The Threshold Autoregressive Stochastic Volatility (TARSV) is then an extension of the ordinary symmetric SARV model. The threshold specification in TARSV model allows the mean and variance to response differently to the previous values of the time series. See Breidt (1996) and Lam et al. (2002).

The object of this paper is the determination of the tail behavior of the logvolatility process.

The exposition proceeds as follows. Section 2 describes the threshold autoregressive stochastic volatility (TARSV) model and specific conditions for strict stationarity of the log-volatility process are provided. Section 3 contains the tail behavior of the log-volatility process $\left(\alpha_{t}\right)_{t}$. The marginal distribution of $\left(\alpha_{t}\right)_{t}$ has Pareto-like tails.

\section{The model}

The threshold autoregressive stochastic volatility (TARSV) model introduced by Breidt (1996) for $Y_{t}$ is defined by the following relation

$$
Y_{t}=\sigma \exp \left(\frac{\alpha_{t}}{2}\right) \varepsilon_{t}
$$

where $\left(\alpha_{t}\right)_{t}$ is an open-loop threshold autoregressive process (Tong 1990, p. 101),

$$
\alpha_{t}= \begin{cases}\phi_{1} \alpha_{t-1}+Z_{t}^{(1)}, & \text { if } Y_{t-1} \leq 0 \\ \phi_{2} \alpha_{t-1}+Z_{t}^{(2)}, & \text { if } Y_{t-1}>0\end{cases}
$$

where $\phi_{i}$ are non-random constants and with threshold variable $Y_{t-1}$. In this framework, $\sigma$ is a positive constant and $\left(\varepsilon_{t}\right)_{t}$ is a sequence of independent and identically distributed random variables with zero mean and its variance is taken to be one. The log-volatility process has a piecewise linear structure. It switches between two first-order autoregessive processes according to the sign of the previous observation.

We will use the following assumptions 
- $H_{1}:\left(Z_{t}^{(i)}\right)$ is a sequence of independent and identically distributed random variables $(i=1,2)$ and satisfies the following conditions :

$$
E\left[\log ^{+}\left|Z_{1}^{(i)}\right|\right]<+\infty
$$

where $\log ^{+} x=\max (0, \log x)$.

- $H_{2}$ : For each $i=1,2$, the two sequences of random variables $\left(Z_{t}^{(i)}\right)_{t}$ and $\left(\varepsilon_{t}\right)_{t}$ are independent and $\left(Z_{t}^{(1)}\right)_{t}$ and $\left(Z_{t}^{(2)}\right)_{t}$ are independent.

- $H_{3}$ : The process $\left(Z_{t}^{(i)}\right)_{t}$ has a heavy tailed structure, that is

$$
\mathbb{P}\left\{\left|Z_{1}^{(i)}\right|>x\right\}=x^{-\alpha} L_{i}(x), \quad \alpha>0, \quad i=1,2
$$

and

$$
\lim _{x \rightarrow \infty} \frac{\mathbb{P}\left\{Z_{1}^{(i)}>x\right\}}{\mathbb{P}\left\{\left|Z_{1}^{(i)}\right|>x\right\}}=p, \quad \lim _{x \rightarrow \infty} \frac{\mathbb{P}\left\{Z_{1}^{(i)}<-x\right\}}{\mathbb{P}\left\{\left|Z_{1}^{(i)}\right|>x\right\}}=1-p,
$$

where $L_{i}$ is a slowly varying function at $\infty$ and $0 \leq p \leq 1$.

We define $q=\mathbb{P}\left\{\varepsilon_{t} \leq 0\right\}$ and $I_{1 t}=\mathbf{1}_{\left\{Y_{t-1} \leq 0\right\}}, I_{2 t}=1-I_{1 t}$. Then the equation (2.2) can be rewritten as :

$$
\alpha_{t}=\phi_{(t)} \alpha_{t-1}+Z_{t}
$$

where

$$
\phi_{(t)}=\phi_{1} I_{1 t}+\phi_{2} I_{2 t} \quad \text { and } \quad Z_{t}=Z_{t}^{(1)} I_{1 t}+Z_{t}^{(2)} I_{2 t} .
$$

The equation (2.6) is a stochastic difference equation where the pairs $\left(\phi_{(t)}, Z_{t}\right)_{t}$ are sequences of independent identically distributed $\mathbb{R}^{2}$-valued random variables under $\mathrm{H} 1-\mathrm{H} 2$.

The next proposition gives the strict stationarity of the process $\left(\alpha_{t}\right)_{t}$ defined in (2.6). This result follows from Theorem 1 of Brandt (1986).

Proposition 1 Assume that the assumptions $H_{1}$ and $H_{2}$ are specified and that $\left|\phi_{1}\right|^{q}\left|\phi_{2}\right|^{1-q}<1$. Then, for all $t \in \mathbb{Z}$, the series $\left(\alpha_{t}\right)_{t}$ defined in (2.6) admits the following expansion

$$
\alpha_{t}=\sum_{j=0}^{\infty}\left(\prod_{k=0}^{j-1} \phi_{(t-k)}\right) Z_{t-j}
$$

which converges almost surely. Then the process $\left(\alpha_{t}\right)_{t}$ is the unique strictly stationary solution of (2.6). 
From the equations (2.4) and (2.5), we check that the random variables $Z_{k}$ have regularly varying tails; that is,

$$
\mathbb{P}\left\{\left|Z_{k}\right|>x\right\}=x^{-\alpha} L(x), \quad x>0
$$

where $L=q L_{1}+(1-q) L_{2}$ is a slowly varying function at $\infty$ and

$$
\lim _{x \rightarrow \infty} \frac{\mathbb{P}\left\{Z_{k}>x\right\}}{\left.\mathbb{P}\left\{\left|Z_{k}\right|>x\right\}\right]}=p, \quad \lim _{x \rightarrow \infty} \frac{\mathbb{P}\left\{Z_{k}<-x\right\}}{\mathbb{P}\left\{\left|Z_{k}\right|>x\right\}}=1-p .
$$

An important class of distributions satisfying (2.8) and (2.9) consists of the non-normal stable distributions. For an extensive discussion of stable random variables see Feller (1971), pp. 568-583 and Samorodnitsky and Taqqu (1994).

\section{Tail behavior of the stationary distribution of the TAR model}

In this section we study the tail behavior of the distribution of the process $\left(\alpha_{t}\right)_{t}$ defined in (2.6). The tail behavior of the stationary solution of such equations has been widely studied in a variety of contexts. See for example Kesten (1973), Grincevicius (1975), Vervaat (1979), Resnick and Willekens (1991). In these papers, either the multiplier in the stochastic difference equation is a positive random coefficient or the noise term is an iid $\mathbb{R}^{+}$-valued random variable. Furthermore, in general, the coefficient and the noise are assumed to be independent. However the random coefficient model (2.6) does not necessarily satisfy the positivity condition on the multiplier $\phi_{(t)}$ and the noise term $\left(Z_{t}\right)_{t}$. In our framework, the model (2.7) can be seen as a moving average with random coefficients and driven by regularly varying noise according to (2.8) and (2.9). Note that the coefficient and the noise term are dependent.

We prove in the following result that both the right and the left tails of the marginal distribution of $\left(\alpha_{t}\right)_{t}$ are regularly varying with tail exponent $-\alpha$ with $\alpha>0$. We also determine the exact values of the coefficients in the tail behaviour of the stationary solution of the stochastic difference equation (2.6).

Theorem 1 Let $\left(\alpha_{t}\right)_{t}$ be the stationary solution of the equation (2.6) and the process $\left(Z_{t}\right)_{t}$ satisfying (2.8) and (2.9). Suppose that the assumptions of Proposition 1 hold. Furthermore, if $q\left|\phi_{1}\right|^{\alpha}+(1-q)\left|\phi_{2}\right|^{\alpha}<1$, then the tail behavior of the stationary distribution of $\left(\alpha_{t}\right)_{t}$ is :

$$
\mathbb{P}\left\{\alpha_{t}>x\right\} \sim\left(\sum_{k=0}^{\infty} C_{k}(p, \alpha)\right) x^{-\alpha} L(x), \text { as } x \rightarrow \infty,
$$


where

$C_{k}(p, \alpha)=\left\{\begin{aligned} p E\left|\phi_{(1)}\right|^{k \alpha} & \text { if } \quad \phi_{1}>0, \phi_{2}>0, \\ \left(p \delta_{k}+(1-p) \delta_{k+1}\right) E\left|\phi_{(1)}\right|^{k \alpha} & \text { if } \quad \phi_{1}<0, \phi_{2}<0, \\ \sum_{i=0}^{k}\left(p \delta_{i}+(1-p) \delta_{i+1}\right) \mathrm{C}_{k}^{i}\left(q\left|\phi_{1}\right|^{\alpha}\right)^{k-i}\left((1-q)\left|\phi_{2}\right|^{\alpha}\right)^{i} & \text { if } \quad \phi_{1}>0, \phi_{2}<0, \\ \sum_{i=0}^{k}\left(p \delta_{i}+(1-p) \delta_{i+1}\right) \mathrm{C}_{k}^{i}\left(q\left|\phi_{1}\right|^{\alpha}\right)^{i}\left((1-q)\left|\phi_{2}\right|^{\alpha}\right)^{k-i} & \text { if } \quad \phi_{1}<0, \phi_{2}>0 .\end{aligned}\right.$

and

$$
\mathbb{P}\left\{\alpha_{t}<-x\right\} \sim\left(\sum_{k=0}^{\infty} C_{k}(1-p, \alpha)\right) x^{-\alpha} L(x), \text { as } x \rightarrow \infty,
$$

with

$$
\delta_{i}= \begin{cases}1 & \text { if } i \text { even } \\ 0 & \text { if } i \text { odd }\end{cases}
$$

In addition, we have,

$$
\mathbb{P}\left\{\left|\alpha_{t}\right|>x\right\} \sim\left(\sum_{k=0}^{\infty} E\left|\phi_{(1)}\right|^{k \alpha}\right) x^{-\alpha} L(x), \text { as } x \rightarrow \infty .
$$

Before proving Theorem 1 we establish two lemmas.

Lemma 1 Let $\psi_{1}$ a random variable independent of $Z_{1}$ and $\phi_{(1)}$ such that

$$
\lim _{x \rightarrow \infty} \frac{\mathbb{P}\left(\psi_{1}>x\right)}{x^{-\alpha} L(x)}=C \quad \text { and } \quad \lim _{x \rightarrow \infty} \frac{\mathbb{P}\left(\psi_{1}<-x\right)}{x^{-\alpha} L(x)}=C^{\prime},
$$

where $C$ and $C^{\prime}$ are positive constants. Then

$$
\mathbb{P}\left(Z_{1}+\phi_{(1)} \psi_{1}>x\right) \sim\left(p+K\left(\alpha, C, C^{\prime}\right)\right) x^{-\alpha} L(x), \quad \text { as } \quad x \rightarrow \infty
$$

and

$$
\mathbb{P}\left(Z_{1}+\phi_{(1)} \psi_{1}<-x\right) \sim\left(1-p+K\left(\alpha, C^{\prime}, C\right)\right) x^{-\alpha} L(x), \quad \text { as } \quad x \rightarrow \infty
$$

where

$$
K\left(\alpha, C, C^{\prime}\right)=\left\{\begin{array}{rll}
C E\left|\phi_{(1)}\right|^{\alpha} & \text { if } & \phi_{1}>0, \phi_{2}>0, \\
C^{\prime} E\left|\phi_{(1)}\right|^{\alpha} & \text { if } & \phi_{1}<0, \phi_{2}<0, \\
q C\left|\phi_{1}\right|^{\alpha}+(1-q) C^{\prime}\left|\phi_{2}\right|^{\alpha} & \text { if } & \phi_{1}>0, \phi_{2}<0, \\
q C^{\prime}\left|\phi_{1}\right|^{\alpha}+(1-q) C\left|\phi_{2}\right|^{\alpha} & \text { if } & \phi_{1}<0, \phi_{2}>0 .
\end{array}\right.
$$




\section{Proof :}

From (3.4), it is easily checked that

$$
\lim _{x \rightarrow+\infty} \frac{\mathbb{P}\left(\phi_{(1)} \psi_{1}>x\right)}{x^{-\alpha} L(x)}=K\left(\alpha, C, C^{\prime}\right)
$$

and

$$
\lim _{x \rightarrow+\infty} \frac{\mathbb{P}\left(\phi_{(1)} \psi_{1}<-x\right)}{x^{-\alpha} L(x)}=K\left(\alpha, C^{\prime}, C\right) .
$$

First we shall prove assertion (3.5). For $0<\varepsilon<1$ and $x>1$, we have

$$
\begin{aligned}
\mathbb{P}\left\{Z_{1}+\phi_{(1)} \psi_{1}>x\right\} & \geq \mathbb{P}\left\{Z_{1}>x(1+\varepsilon),\left|\phi_{(1)} \psi_{1}\right|<x \varepsilon\right\}+\mathbb{P}\left\{\left|Z_{1}\right|<x \varepsilon, \phi_{(1)} \psi_{1}>x(1+\varepsilon)\right\} \\
& \geq \mathbb{P}\left\{Z_{1}>x(1+\varepsilon)\right\}+\mathbb{P}\left\{\phi_{(1)} \psi_{1}>x(1+\varepsilon)\right\} \\
-\mathbb{P}\left\{Z_{1}\right. & \left.>x(1+\varepsilon),\left|\phi_{(1)} \psi_{1}\right| \geq x \varepsilon\right\}-\mathbb{P}\left\{\left|Z_{1}\right|>x \varepsilon, \phi_{(1)} \psi_{1}>x(1+\varepsilon)\right\} .
\end{aligned}
$$

For all $\beta$ such that $\beta-\alpha>0$ the first term of (3.10) can be written as $\mathbb{P}\left\{Z_{1}>x(1+\varepsilon),\left|\phi_{(1)} \psi_{1}\right|>x \varepsilon\right\} \leq \mathbb{P}\left\{\left|\phi_{(1)}\right|>x^{\frac{\alpha+\beta}{2 \beta}}\right\}+\mathbb{P}\left\{Z_{1}>x(1+\varepsilon)\right\} \mathbb{P}\left\{\left|\psi_{1}\right|>x^{\frac{\beta-\alpha}{2 \beta}} \varepsilon\right\}$.

By the Markov's inequality it follows that

$$
\frac{\mathbb{P}\left\{\left|\phi_{(1)}\right|>x^{\frac{\alpha+\beta}{2 \beta}}\right\}}{x^{-\alpha} L(x)} \leq \frac{E\left|\phi_{(1)}\right|^{\beta}}{x^{\beta} L(x)}
$$

which goes to 0 as $x \rightarrow \infty$. By (2.8) and (2.9), we get

$$
\lim _{x \rightarrow+\infty} \frac{\mathbb{P}\left\{Z_{1}>x(1+\varepsilon)\right\} \mathbb{P}\left\{\left|\psi_{1}\right|>x^{\frac{\beta-\alpha}{2 \beta}} \varepsilon\right\}}{x^{-\alpha} L(x)}=0 .
$$

Hence

$$
\frac{\mathbb{P}\left\{Z_{1}>x(1+\varepsilon),\left|\phi_{(1)} \psi_{1}\right|>x \varepsilon\right\}}{x^{-\alpha} L(x)} \rightarrow 0 \text { as } x \rightarrow \infty .
$$

Analogously the second term of (3.10) is handled very similarly as the first one

$$
\frac{\mathbb{P}\left\{\left|Z_{1}\right|>x \varepsilon, \phi_{(1)} \psi_{1}>x(1+\varepsilon)\right\}}{x^{-\alpha} L(x)} \rightarrow 0 \text { as } x \rightarrow \infty .
$$

Finally by (2.8), (2.9), (3.8),(3.11) and (3.12), we have

$$
\lim _{x \rightarrow \infty} \frac{\mathbb{P}\left(Z_{1}+\phi_{(1)} \psi_{1}>x\right)}{x^{-\alpha} L(x)} \geq p+K\left(\alpha, C, C^{\prime}\right) .
$$

On the other hand,

$\mathbb{P}\left\{Z_{1}+\phi_{(1)} \psi_{1}>x\right\} \leq \mathbb{P}\left\{Z_{1}>x(1-\varepsilon)\right\}+\mathbb{P}\left\{\phi_{(1)} \psi_{1}>x(1-\varepsilon)\right\}+\mathbb{P}\left\{Z_{1}>x \varepsilon, \phi_{(1)} \psi_{1}>x \varepsilon\right\}$.

Using the independence between the random variables $\psi_{1}$ and $Z_{1}$, the second term of the right-hand side of (3.14) gives

$\mathbb{P}\left(Z_{1}>x \varepsilon, \phi_{(1)} \psi_{1}>x \varepsilon\right)=q \mathbb{P}\left(Z_{1}>x \varepsilon\right) \mathbb{P}\left(\phi_{1} \psi_{1}>x \varepsilon\right)+(1-q) \mathbb{P}\left(Z_{1}>x \varepsilon\right) \mathbb{P}\left(\phi_{2} \psi_{1}>x \varepsilon\right)$. 
Therefore

$$
\begin{aligned}
\lim _{x \rightarrow \infty} \frac{\mathbb{P}\left(Z_{1}>x \varepsilon, \phi_{(1)} \psi_{1}>x \varepsilon\right)}{x^{-\alpha} L(x)} & =\lim _{x \rightarrow \infty}\left[q p \varepsilon^{-\alpha} \mathbb{P}\left(\phi_{1} \psi_{1}>x \varepsilon\right)+(1-q) p \varepsilon^{-\alpha} \mathbb{P}\left(\phi_{2} \psi_{1}>x \varepsilon\right)\right] \\
& =0 .
\end{aligned}
$$

Thus by (2.8), (2.9), (3.8) and (3.15), we have

$$
\lim \sup _{x \rightarrow \infty} \frac{\mathbb{P}\left(Z_{1}+\phi_{(1)} \psi_{1}>x\right)}{x^{-\alpha} L(x)} \leq p+K\left(\alpha, C, C^{\prime}\right)
$$

Combining (3.13) and (3.16), we obtain (3.5).

The proof of (3.6) is achieved similarly and need (3.9).

Lemma 2 Suppose $E\left|\phi_{(1)}\right|^{\alpha}<1$. For all b such that $1<b^{\alpha}<\frac{1}{E\left|\phi_{(1)}\right|^{\alpha}}$ and for all $n \geq 1$, we have

$\lim _{x \rightarrow \infty} \frac{\mathbb{P}\left\{\sum_{j=n+1}^{\infty}\left(\prod_{k=0}^{j-1} \phi_{(t-k)}\right) Z_{t-j}>x \varepsilon\right\}}{x^{-\alpha} L(x)} \leq \frac{1}{\left((b-1) \varepsilon b^{n}\right)^{\alpha}} \sum_{k=n+1}^{\infty}\left(E\left|\phi_{(1)}\right|^{\alpha} b^{\alpha}\right)^{k}$.

\section{Proof :}

First observe that

$$
\begin{array}{r}
\mathbb{P}\left\{\sum_{j=n+1}^{\infty}\left(\prod_{k=0}^{j-1} \phi_{(t-k)}\right) Z_{t-j}>x \varepsilon\right\} \leq \mathbb{P}\left\{\sum_{j=n+1}^{\infty}\left|\prod_{k=0}^{j-1} \phi_{(t-k)}\right|\left|Z_{t-j}\right|>x \varepsilon\right\} \\
\leq \sum_{j=n+1}^{\infty} \mathbb{P}\left\{\left|\phi_{(t)} \phi_{(t-1)} \ldots \phi_{(t-j+1)}\right|\left|Z_{t-j}\right|>b^{n-j}(b-1) x \varepsilon\right\} \\
\leq \sum_{j=n+1}^{\infty} \sum_{i=0}^{j} \mathbb{P}\left\{\left|\phi_{1}^{i} \phi_{2}^{j-i}\right|\left|Z_{t-j}\right|>b^{n-j}(b-1) x \varepsilon\right\} p_{i}
\end{array}
$$

where $p_{i}=\mathrm{C}_{j}^{i} q^{i}(1-q)^{j-i}$. From (2.8) and (2.9), we get

$$
\lim _{x \rightarrow+\infty} \frac{\sum_{i=0}^{j} \mathbb{P}\left\{\left|\phi_{1}^{i} \phi_{2}^{j-i}\right|\left|Z_{t-j}\right|>b^{n-j}(b-1) x \varepsilon\right\} p_{i}}{x^{-\alpha} L(x)} \leq\left(\varepsilon b^{n}(b-1)\right)^{-\alpha}\left(E\left|\phi_{(1)}\right|^{\alpha} b^{\alpha}\right)^{j} .
$$

This implies the assertion.

\section{Proof of Theorem 1:}

By induction of the result stated in Lemma 1, we have

$$
\mathbb{P}\left\{\sum_{j=0}^{n}\left(\prod_{k=0}^{j-1} \phi_{(t-k)}\right) Z_{t-j}>x\right\} \sim\left(\sum_{k=0}^{n} C_{k}(p, \alpha)\right) x^{-\alpha} L(x),
$$


as $x \rightarrow \infty$.

First, we shall show that

$$
\lim _{x \rightarrow \infty} \frac{\mathbb{P}\left\{\sum_{j=0}^{\infty}\left(\prod_{k=0}^{j-1} \phi_{(t-k)}\right) Z_{t-j}>x\right\}}{x^{-\alpha} L(x)} \geq \sum_{k=0}^{\infty} C_{k}(p, \alpha) .
$$

Set

$$
S_{n}=\sum_{j=0}^{n}\left(\prod_{k=0}^{j-1} \phi_{(t-k)}\right) Z_{t-j} \quad \text { and } \quad R_{n}=\sum_{j=n+1}^{\infty}\left(\prod_{k=0}^{j-1} \phi_{(t-k)}\right) Z_{t-j} .
$$

Notice that

$$
\begin{aligned}
\mathbb{P}\left\{S_{n}+R_{n}>x\right\} & \geq \mathbb{P}\left\{S_{n}>x(1+\varepsilon),\left|R_{n}\right|<x \varepsilon\right\} \\
& \geq \mathbb{P}\left\{S_{n}>x(1+\varepsilon)\right\}-\mathbb{P}\left\{\left|R_{n}\right| \geq x \varepsilon\right\} .
\end{aligned}
$$

Using lemma 2 and (3.18), we have for all $n \geq 1$ and $\varepsilon>0$,

$\lim _{x \rightarrow \infty} \frac{\mathbb{P}\left\{S_{n}+R_{n}>x\right\}}{x^{-\alpha} L(x)} \geq\left(\sum_{k=0}^{n} C_{k}(p, \alpha)\right)(1+\varepsilon)^{-\alpha}-\frac{1}{\left((b-1) \varepsilon b^{n}\right)^{\alpha}} \sum_{k=n+1}^{\infty}\left(E\left|\phi_{(1)}\right|^{\alpha} b^{\alpha}\right)^{k}$,

and from this inequality, (3.19) follows.

On the other hand, we have

$$
\mathbb{P}\left\{S_{n}+R_{n}>x\right\} \leq \mathbb{P}\left\{S_{n}>x(1-\varepsilon)\right\}+\mathbb{P}\left\{R_{n}>x \varepsilon\right\} .
$$

Then using again lemma 2 and (3.18), we get

$$
\lim \sup _{x \rightarrow \infty} \frac{\mathbb{P}\left\{S_{n}+R_{n}>x\right\}}{x^{-\alpha} L(x)} \leq \sum_{k=0}^{\infty} C_{k}(p, \alpha) .
$$

Combining (3.19) and (3.20), we obtain the desired result. The theorem is entirely demonstrated.

\section{References}

Andersen, T. G., (1994). Stochastic autoregressive volatility : a framework for volatility modeling. Mathematical finance, 4 (2), 75-102.

Brandt, A. (1986). The stochastic equation $Y_{n+1}=A_{n} Y_{n}+B_{n}$ with stationary coefficients. Adv. Appl. Probab. 18, 211-220.

Breidt, F.J. (1996). A Threshold Autoregressive Stochastic Volatility Model. VI Latin American Congress of Probability and Mathematical Statistics (CLAPEM), 
Valparaiso, Chile.

Clark, P.K. (1973). A subordinated stochastic process model with finite variances for speculative prices, Econometrica, 41, 135-156.

Fama, E. (1965). Behavior of stock market prices, J. Bus. U. Chicago, 38, $34-105$.

Feller, W. (1971). An Introduction to the theory of Probability and Its Applications, Vol. 2, 2nd ed., John Wiley, New York.

Grincevicius, A. (1975). On a limit distribution for a random walk on the line. Lithuanian Math. J., 15, 580-589.

Kesten, H. (1973). Random difference equations and renewal theory for products of random matrices. Acta. Math., 131, 207-248.

Lam, K., Li, W.K. and So, M.K.P. (2002). A Threshold Stochastic Volatility model, Journal of Forecasting, 21, 473-500.

Nelson, D. B., (1991). Conditional Heteroskedasticity in Asset Returns : A new Approach. Econometrica, 59, 347-370.

Resnick, S.I. and Willekens, E. (1991). Moving averages with random coefficients and random coefficient autoregressive models. Stochastic Models, 7(4), $511-525$.

Samorodnitsky, G. and Taqqu, M.S. (1994). Stable Non-Gaussian Random Processes. Stochastic Models with Infinite Variance. Chapman and Hall, London.

Stuck, B.W. and Kleiner, B. (1974). A statistical analysis of telephone noise, The Bell System Technical Journal, 53, 1263-1320.

Taylor, S., (1986). Modelling Financial Time series. Wiley, New York.

Taylor, S., (1994). Modeling Stochastic Volatility : A Review and Comparative Study. Mathematical Finance, 4, 183-204. 
Tong, H. (1990). Non-linear Time series : A Dynamical System Approach, Oxford University Press, New York.

Vervaat, W. (1979). On a stochastic difference equation and a representation of non-negative infinitely divisible random variables. Adv. Appl. Probab., 11, 750-783. 